\title{
REPAssistive: um Repositório de Recursos Digitais para Educandos com Necessidades Educacionais Específicas
}

\author{
José Vinícius Vieira Lima1, Ellen Polliana R. Souza2, Ana Carolina C. de Meloz \\ 1Universidade de Pernambuco - (UPE) \\ ${ }_{2}$ Universidade Federal Rural de Pernambuco - (UFRPE). \\ jvv@ecomp.poli.br, \{ellen.ramos, carolina.melo\}@ufrpe.br
}

\begin{abstract}
Digital Educational Resources have been used as tools which assist students with disabilities in the learning process. However, educators have difficulty to locate such resources in a cataloged way and according to the type of the learner disability. The aim of this work is to present the development of a digital repository with specific educational games for special education using the accessibility guidelines for WEB content. In this way, the repository has been successfully evaluated and it is composed by: a collaborative space, advanced search engine and voice, besides the catalog of games together with the set of technical and pedagogical information present in the teacher's manual.
\end{abstract}

Resumo. Recursos Educacionais Digitais têm sido utilizados como ferramentas que auxiliam os educandos com deficiência no processo de aprendizagem. Porém, há uma dificuldade por parte dos educadores em localizá-los de forma catalogada e de acordo com o tipo de deficiência. O objetivo deste trabalho é apresentar o desenvolvimento de um repositório digital com jogos educacionais específicos para a educação especial utilizando as diretrizes de acessibilidade para o conteúdo WEB. Deste modo, o repositório foi avaliado com sucesso, o mesmo dispõe de um espaço colaborativo, mecanismo de busca avançada e por voz, além do catálogo de jogos, como também o conjunto de informações técnicas e pedagógicas presentes no manual do professor.

\section{Introdução}

O termo Pessoas com Necessidades Educacionais Específicas (NEE) refere-se a todos os educandos que apresentam, em caráter temporário ou permanentemente, significativas diferenças físicas, sensoriais ou intelectuais, cujas fragilidades decorrem de fatores naturais ou adquiridos, acarretando em dificuldades na interação com a sociedade e, no modo como aprendem [Silva et al. 2011]. As NEE estão incluídas no que caracteriza-se como Educação Especial. Sendo esta, uma área que promove práticas relacionadas ao ambiente pedagógico, garantindo a inclusão por meio de instrumentos que promovam vantagens em relação à melhoria do desempenho intelectual e cognitivo.

As Pessoas com Deficiência (PcD), frequentemente, enfrentam obstáculos no seu desenvolvimento e comportamento, principalmente no aspecto da adequação no ambiente escolar. Segundo Silva et al. (2011), a falta de acesso a tecnologias traz 
prejuízos consideráveis ao indivíduo, limitando sua capacidade produtiva e também a sua cidadania. Desta maneira, Lima et al. (2016) relatam que muitas práticas em prol de pessoas com NEE vêm sendo aderidas, buscando a promoção de igualdades com seus pares e um melhor convívio no meio pedagógico e social.

Para uma melhor relação diante da sociedade e técnicas que minimizem, desenvolvam ou superem às dificuldades potencializadoras do aprendizado em educandos com NEE, os jogos educacionais digitais se destacam como uma eficiente ferramenta pedagógica [Silva et al. 2011]. Do mesmo modo, Valente et al. (2018) mencionam que esses jogos, frequentemente, estão alinhados com teorias de aprendizagem, pressupondo uma aprendizagem dinâmica, ativa, contextualizada e baseada em problemas. Esse tipo de recurso, destaca-se como método que promove o ensino e aprendizagem por meio da aplicação de componentes curriculares, incentivando a atenção e concentração.

Os jogos digitais podem ser encontrados em os repositórios virtuais, que também são conhecidos como portais educacionais. Estes, oferecem ao usuário, em uma página $W E B$, diversas opções de Recursos Educacionais Digitais (RED) a serem incorporados de acordo com o currículo escolar e os diferentes níveis de educação [Silva et al. 2016]. Considerando esse contexto, atualmente, os repositórios educacionais existentes não possuem um espaço específico e destinado à educação especial e educandos com NEE, o que dificulta a busca de RED pelos educadores que desejam aplicá-los dentro da sala de aula.

Assim, este trabalho tem como objetivo apresentar o desenvolvimento de um repositório de RED, inicialmente apenas com jogos que tenham características próprias para o uso e partilha de conteúdos relacionados à linguagem e ao raciocínio lógico, nos quais são direcionados para educandos com NEE. Como apoio neste processo, os requisitos foram identificados e validados por educadores da Associação de Pais e Amigos dos Excepcionais (APAE) de Serra Talhada - PE.

O trabalho está estruturado da seguinte forma: a Seção 2 apresenta o referencial teórico que embasará as reflexões sobre a educação especial, o uso de jogos educacionais por educandos com NEE, a importância dos repositórios educacionais, e os trabalhos relacionados. A Seção 3 compõe os procedimentos metodológicos e as ferramentas utilizadas no desenvolvimento do repositório. Na Seção 4, é apresentado o REPAssistive e suas funcionalidades. A Seção 5 exibe os resultados dos processos de validação. E, por fim, a Seção 6 que discorre sobre as considerações finais, contribuições e trabalhos futuros.

\section{Referencial Teórico}

\subsection{Educação Especial}

Educação Especial constitui-se como modalidade de ensino oferecida na educação escolar, de preferência, na rede regular, visando promover o desenvolvimento das potencialidades de pessoas com NEE [Lima et al. 2016]. Conforme Carvalho (2010), a prática da educação está estritamente associada à inclusão, sendo compreendida como uma metodologia que exerce o direito de igualdade e equidade de oportunidades.

A mudança do paradigma da integração para o modelo de inclusão, reflete, principalmente no âmbito educacional em conformidade com a preocupação em atender 
à diversidade. As transformações dos sistemas educacionais estão associadas à construção de um novo tipo de sociedade, através da inclusão gerada com o uso da educação, tornando-se um conceito revolucionário que busca promover as barreiras que mantém a exclusão em seu sentido mais lato e pleno.

Aprovada em 2015, a Lei Brasileira de Inclusão (LBI), de $\mathrm{n}^{0} 13.146$, conta com mais de 100 artigos voltados a promoção da igualdade e oportunidades para $\mathrm{PcD}$. A Lei se insere na comunidade escolar, provocando a garantia do ensino de qualidade à pessoa com deficiência, colocando-a a salva de toda forma de violência, negligência e discriminação. Assegurando também o acesso a produtos, recursos, práticas, métodos e processos que tendem maximizar a autonomia, mobilidade pessoal e qualidade de vida.

\subsection{Jogos Educacionais Digitais}

Os jogos educacionais digitais recebem destaque como instrumento de amplo auxílio para a educação especial, oferecendo aos usuários a perspectiva de exercitar os seus conhecimentos em cenários virtuais que permitem uma maior interatividade, tentativa e erros, considerando o seu tempo cognitivo, potencializando as habilidades e competências que viabilizam a prática do aprendizado. $\mathrm{O}$ modo como os usuários interagem com esses jogos, proporciona uma importante alternativa que fortalece $o$ processo de inclusão social diante dos recursos que potencializam-se dentro do ambiente educacional [Lima et al. 2016].

De acordo com Valente et al. (2018), a aprendizagem baseada em jogos é uma forma de engajar os educandos em atividades significativas e permitir que assumam novas identidades, explorem as oportunidades e elevem o aprendizado. Desta forma, esse tipo de recurso tem sido utilizado como motivação de incentivo a concentração, coordenação motora e memorização aos educandos com NEE quando realizam o seu uso, contribuindo assim para uma maior fixação dos conteúdos trabalhados.

\subsection{Repositórios de Recursos Educacionais Digitais}

Repositórios educacionais são importantes recursos que possibilitam aos usuários uma forma detalhada e eficaz de pesquisa. Um repositório deve ser capaz de oferecer um ambiente colaborativo para o desenvolvimento, avaliação e partilha de materiais. Além disso, Júnior (2013) descreve que os repositórios devem recuperar os recursos através de palavras-chave, ou a utilização de uma busca avançada que permita detalhar valores, tendo como intuito melhorar o filtro da pesquisa a depender da necessidade.

Segundo Lima et al. (2016), os repositórios educacionais são portas de acesso a outros websites de caráter instrutivo, além de oferecer diversos serviços à comunidade educativa (diretores, educadores, educandos e familiares), dentro de um ambiente WEB que permite promover informação, mecanismo de pesquisa de dados, ferramenta de colaboração, atividades formativas, catálogos de recursos, entretenimento e lazer.

\subsection{Trabalhos Relacionados}

Atualmente, há diversos repositórios educacionais de livre acesso. Isto é, que possuem a Licença Creative Commons, os mesmos contêm uma vasta quantidade de métodos instrucionais entre os níveis educacionais de ensino. São exemplos desses repositórios: o Banco Internacional de Objetos Educacionais (BIOE), a Plataforma do Ministério da 
Educação de Recursos Educacionais Digitais (MEC-RED), a Rede Interativa Virtual de Educação (RIVED) e o Portal do Professor.

Embora tenha sido realizado o acesso detalhado destas plataformas, não foi encontrado nesses principais repositórios um espaço com recursos concentrados para o uso na educação especial, o que ocasiona-se em uma maior dificuldade no processo de busca e seleção por partes dos educadores dessa área. A Tabela 1 detalha os trabalhos relacionados supracitados comparando-os com o repositório desenvolvido neste estudo.

Tabela 1. Visão geral entre os trabalhos relacionados.

\begin{tabular}{|l|l|l|l|l|}
\hline Repositório & \multicolumn{1}{|c|}{$\begin{array}{c}\text { Nível } \\
\text { Educacional }\end{array}$} & $\begin{array}{c}\text { Áea de } \\
\text { Atuação }\end{array}$ & Colaboração & \multicolumn{1}{c|}{$\begin{array}{c}\text { Módulopara } \\
\text { Educaçá Especial }\end{array}$} \\
\hline BIOE & $\begin{array}{l}\text { Educação básica } \\
\text { e superior }\end{array}$ & $\begin{array}{l}\text { Escolar e } \\
\text { profíssional }\end{array}$ & Não possui & Não possui \\
\hline MEC-RED & Educação básica & $\begin{array}{l}\text { Escolar e } \\
\text { profissional }\end{array}$ & Possui & Não possui \\
\hline RIVED & Educação básica & $\begin{array}{l}\text { Escolar e } \\
\text { profissional }\end{array}$ & Não possui & Não possui \\
\hline $\begin{array}{l}\text { Portal do } \\
\text { Professor }\end{array}$ & $\begin{array}{l}\text { Educação básica } \\
\text { e superior }\end{array}$ & Profissional & Possui & Não possui \\
\hline $\begin{array}{l}\text { Este } \\
\text { trabalho }\end{array}$ & $\begin{array}{l}\text { Educaçá } \\
\text { especial }\end{array}$ & $\begin{array}{l}\text { Escolar e } \\
\text { profissional }\end{array}$ & Possui & Possui \\
\hline
\end{tabular}

\section{Procedimento e Método}

O processo de desenvolvimento do repositório foi dividido em quatro etapas. Os detalhes de cada uma destas serão apresentados nas Subseções a seguir.

\subsection{Identificação dos Conteúdos}

Ao utilizar a APAE de Serra Talhada como estudo de caso, foram realizadas entrevistas semi-estruturadas juntamente com a aplicação de um survey contendo seis perguntas para os educadores da Associação, possuindo uma questão de múltipla escolha e as demais de livre formato para resposta textual. Assim, as informações foram analisadas e extraídas, resultando nos principais conteúdos pedagógicos utilizados entre os educadores, sendo classificados em dois tipos: linguagem e raciocínio lógico.

\subsection{Pesquisa e Seleção dos Jogos Educacionais}

Os jogos educacionais selecionados contêm as Diretrizes de Acessibilidade para o Conteúdo Web1 (WCAG) 2.0. Estas oportunizam que os conteúdos possam ser utilizados por um maior número de $\mathrm{PcD}$, incluindo cegueira e baixa visão, surdez e baixa audição, NEE e dificuldades de aprendizagem. Essas normas foram desenvolvidas pelo World Wide Web Consortium (W3C), e a sua estrutura é caracterizada por quatro princípios globais, diretrizes gerais, critérios de sucesso testáveis e um conjunto de técnicas que buscam corresponder às necessidades deste público-alvo.

Como base no apoio das buscas pelos jogos, as Diretrizes Nacionais para Educação Especial na Educação Básica também foram utilizadas. Desta forma, estas auxiliaram na escolha de aspectos técnicos e pedagógicos nesta etapa. Feito isto, os jogos contidos no repositório foram selecionados através de pesquisas feitas na $W E B$, nais quais foram realizadas buscando identificar a presença dos conteúdos apontados pelos educadores, assim como a existência das diretrizes e critérios de acessibilidade. 


\subsection{Desenvolvimento do Repositório}

Com a necessidade em existir um espaço online, no qual as informações e os RED pudessem ser utilizados e compartilhados, o repositório foi desenvolvido no que se caracteriza-se como comunidade aberta, a qual possibilita a inserção de colaborações por parte dos usuários. Desta maneira, o mesmo é mantido sempre ativo, favorecendo o aumento de RED e a troca de conhecimentos entre os próprios usuários que o utilizam.

Neste aspecto, foram utilizadas algumas ferramentas e tecnologias que auxiliaram a construção do repositório. Com isso, utilizou-se a linguagem de programação Python junto com o Django como framework do tipo WEB. Seguindo as recomendações de acessibilidade propostas pelo $\mathrm{W} 3 \mathrm{C}$, a interface gráfica foi desenvolvida utilizando HTML5, em companhia do Bootstrap como framework do tipo CSS. Também, foi utilizada a linguagem de programação JavaScript em conjunto com a jQuery como uma de suas bibliotecas. Como Sistema de Gerenciamento de Banco de Dados, foi empregado o MySQL.

\subsection{Avaliações}

Buscando avaliar o repositório com base na sua acessibilidade e as normas propostas pelo W3C, junto com os jogos que apresentam as diretrizes sugeridas pela WCAG 2.0, um survey foi aplicado com um grupo de cinco educandos com Deficiência Intelectual, sendo dois deles não alfabetizados. Este baseou-se na metodologia de Software Usability Measurement Inventoryz, tendo o objetivo, avaliar a qualidade em relação ao ponto de vista do usuário final, auxiliando na detecção de possíveis falhas. A coleta de dados foi realizada a partir da satisfação, no uso dos elementos educacionais, conteúdos propostos e a forma de como ocorreu a motivação frente às interações realizadas.

Do mesmo modo, um outro procedimento de avaliação foi executado. Neste, buscou-se avaliar o repositório com o apoio em testes de usabilidade junto com o processo de colaboração de novos RED. Para esse fim, um conjunto de 10 heurísticas foram selecionadas e adaptadas de Nielsen (1994). As heurísticas selecionadas, foram:

- H1 - Facilidade na aprendizagem e memorização;

- H2 - Visibilidade do estado do sistema;

- H3 - Estética e design minimalista;

- H4 - Liberdade de controle fácil para o usuário;

- H5 - Equivalência entre o sistema e o mundo real;

- H6 - Consistência e padrões;

- $\quad$ H7 - Flexibilidade e eficiência de uso;

- $\mathbf{H 8}$ - Prevenção de erros;

- H9 - Clareza nas informações;

- H10 - Capacidade de aprender e assimilar de forma intuitiva.

Com isso, outros dois surveys foram aplicados com o propósito de avaliar o valor da gravidade e possíveis problemas detectados entre as heurísticas, cada um contou com 5 perguntas de múltipla escolha baseadas na escala Likert, contendo de 1 a 5 graus de satisfação. O primeiro survey avaliou a opinião de três educadores da Associação, e o outro foi destinado a avaliação de um grupo de cinco especialistas em 
interface gráfica, princípios de design e conceitos de ergonomia de software. Observações diretas também foram utilizadas para coletar informações.

\section{O REPAssistive}

O REPAssistive é um repositório gratuito, educacional, que cataloga, localiza, avalia e compartilha RED de acesso livre para serem aplicados como métodos de ensino e aprendizagem na educação especial. Com isso, o mesmo pode ser utilizado pelos educadores, como também por educandos com NEE. Atualmente, contém jogos educacionais com objetivos pedagógicos relacionados à linguagem e raciocínio lógico e são caracterizados de acordo com os níveis de ensino previstos nas Diretrizes Nacionais para Educação Especial na Educação Básica, sendo eles: Ensino Infantil, Fundamental I e II e Ensino Médio. As principais funcionalidades do repositório incluem:

- Controle de registro (cadastro, visualização, edição e remoção) de RED para a educação especial;

- Gerenciamento de logins (cadastro, visualização, edição e remoção) de usuários;

- Mecanismo de busca avançada e por reconhecimento de voz;

- Manual do professor com a descrição (técnica e pedagógica) do recurso;

- Feedback e pontuação dos RED realizado entre os próprios usuários;

- Espaço destinado para comunicação e dúvidas entre os usuários e o repositório.

Com o propósito relacionado à praticidade no momento do uso, uma interface amigável e fácil de ser utilizada foi desenvolvida. A Figura 1 exibe a página inicial do repositório que conta com um menu na parte superior que dá acesso as demais páginas.

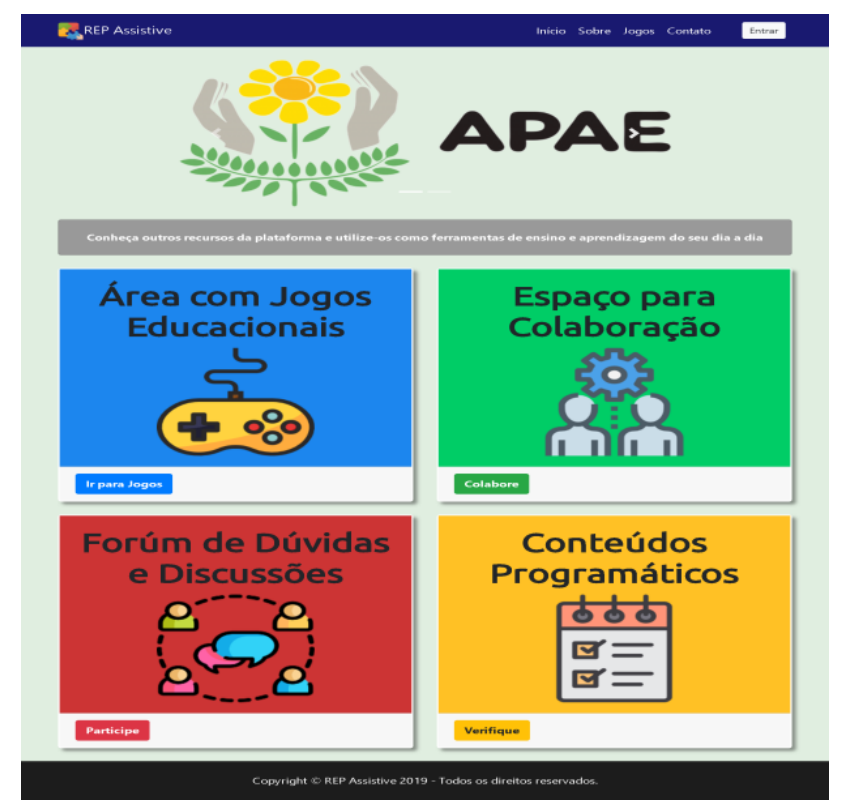

Figura 1. Página inicial do REPAssistive.

Os jogos contidos no repositório dispõem de uma organização que permite identificá-los através do nome que cada um possui, assim como a sua ilustração, na qual facilita a escolha entre os educandos não alfabetizados. Além disso, em cada $c a r d$, é exposto uma breve descrição do jogo, como também o tipo de deficiência que o mesmo se encaixa, qual o seu componente curricular juntamente com o nível de ensino 
apropriado. A Figura 2 apresenta a forma de como os jogos são exibidos e como os mesmos podem ser filtrados.

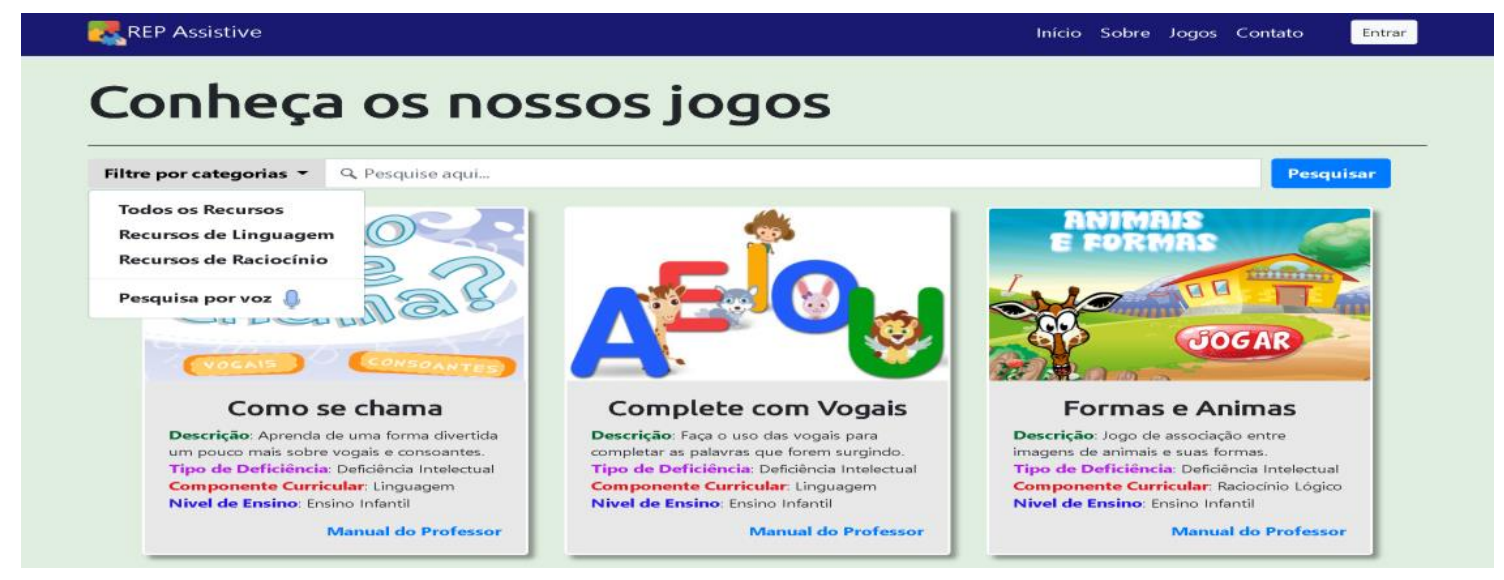

Figura 2. Página de jogos.

As buscas pelos recursos podem ser realizadas por diferentes maneiras, tais como: título, nível educacional, componente curricular, tipo de deficiência ou competências pedagógicas. O repositório também permite que as buscas sejam realizadas utilizando o comando de voz, para utilizá-lo, basta clicar sobre a opção "pesquisa por voz" ou no ícone ilustrado por o símbolo do microfone. Como forma de prover auxílio ao educador, foi desenvolvido o manual do professor. No manual, além das informações já descritas no card do recurso, o mesmo contém a descrição detalhada de cada um deles, incluindo um conjunto de dados pedagógicos e técnicas que contribuem para a preparação e condução da aula. Ao observar a Figura 3, é possível identificar a estrutura do manual e a forma de como as informações são expostas.

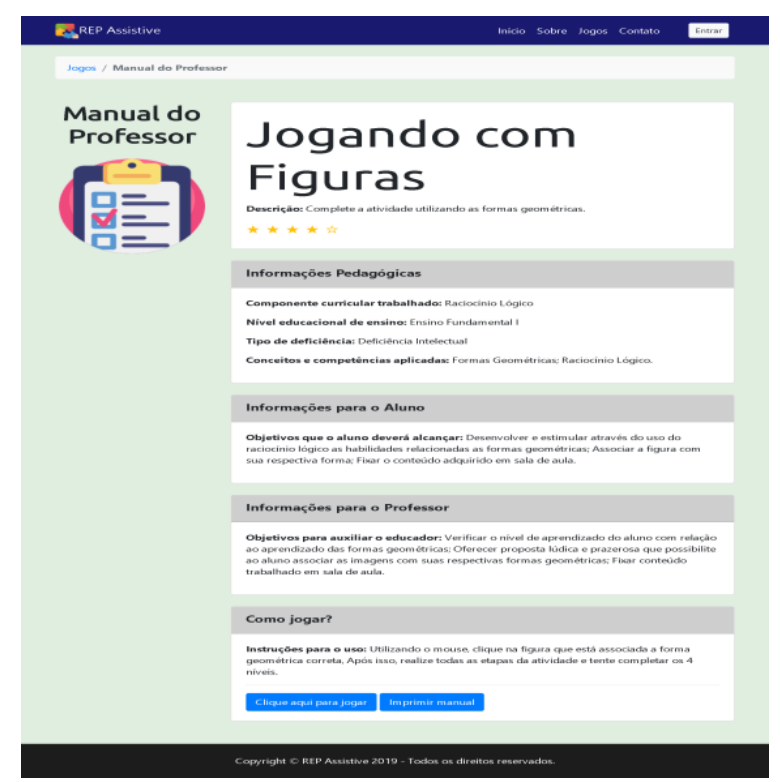

Figura 3. Página do manual do professor.

Dispondo da possibilidade de inserção de novos RED, junto com $o$ fortalecimento da comunidade aberta, o REPAssistive tem como uma das suas principais propostas, um espaço voltado para a colaboração. Em que, proporciona a 
perspectiva do aumento no número de recursos, propagação do conhecimento e a troca de experiência por parte dos usuários. Para realizar uma colaboração, é necessário executar quatro etapas, sendo elas:

1. Criar uma conta no repositório;

2. Preencher os campos destinados com as informações do recurso;

3. Carregar o arquivo, podendo este ser de três tipos: Desktop, WEB ou Mobile;

4. Enviar a proposta.

As propostas enviadas são avaliadas conforme as normas estabelecidas pelo repositório, em que sustentam-se através das recomendações e critérios sugeridos pela WCAG 2.0 junto ao W3C. Desta forma, o usuário tem a opção de visualizar, editar e acompanhar a situação de cada colaboração, na qual possui quatro tipos de status, que são: enviado, em análise, aprovado e recusado. O repositório ainda dispõe de uma página que permite ao usuário interagir, esclarecer dúvidas e enviar sugestões. Com isso, é colocado em prática o suporte em atender às necessidades dos visitantes, promovendo atividades que estimulem o desenvolvimento e melhoramento do mesmo.

\section{Resultados e Discussões}

Os resultados serão discutidos sob dois aspectos: a avaliação dos jogos realizada pelos educandos e a avaliação de usabilidade. Ao fim, as limitações e ameaças são expostas.

\subsection{Avaliação de Acessibilidade dos Jogos}

A avaliação de acessibilidade dos jogos com relação a utilização dos educandos com NEE foi realizada com o propósito de analisar se os jogos cadastrados no repositório são úteis quando associados no processo de aprendizagem e se o repositório desenvolvido pode ser utilizado por estas pessoas. Ao analisar os dados dos surveys respondidos pelos educandos, os resultados apontam que há o estímulo de quatro tipos de habilidades, conforme mostra a Figura 4.

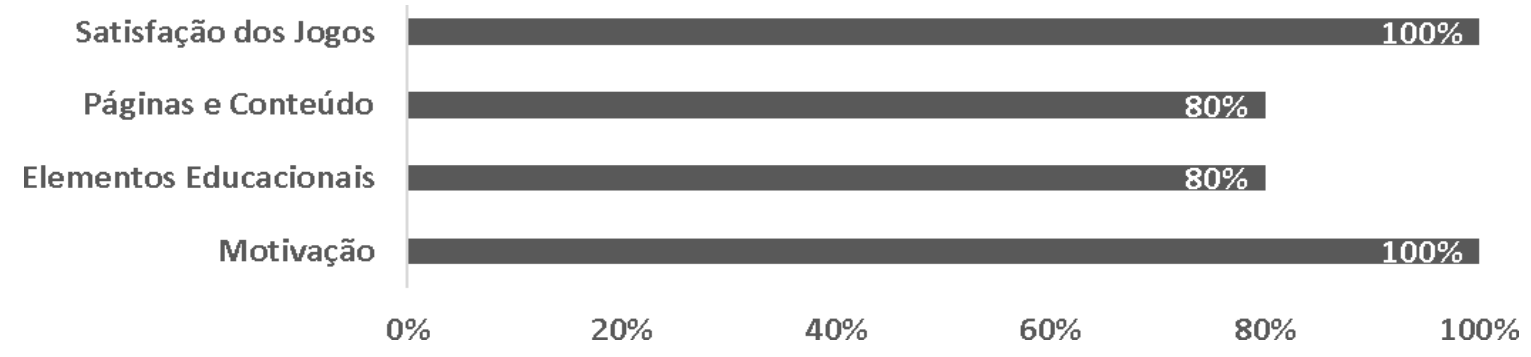

Figura 4. Avaliação das habilidades encontradas nos jogos.

Dentre essas habilidades, é possível observar que 100\% dos educandos responderam que conseguiram utilizar os jogos de modo satisfatório. Da mesma forma que o item relacionado a motivação também obteve aprovação máxima, já que os educandos afirmaram que jogariam outras vezes os jogos contidos no repositório. Esse resultado já era esperado, pois durante a aplicação do survey, foi observado que os mesmos se mostraram motivados em explorar os jogos, bem como a permanência na sala após o fim da avaliação. Em paralelo, apenas um entre o total de avaliados não classificou como "excelente" a forma no qual os conteúdos e as páginas $W E B$ foram configuradas. Assim como os elementos educacionais presentes nos jogos também 
foram medidos com $80 \%$ de aceitação conforme as contribuições que estes oferecem no processo de aprendizagem.

Partindo dos dados apresentados, percebe-se que os educandos avaliaram como conceito máximo as habilidades relacionadas a satisfação dos jogos e a motivação que os mesmos oferecem. Esses resultados correlacionam-se ao fato de que esse tipo de RED, aplicado no processo de aprendizagem, oferece novas formas didáticas e eficazes de adquirir o conhecimento. Desta forma, o modo como os conceitos foram expostos nas páginas, juntamente com os elementos educacionais presentes nos jogos, destacamse como aspectos positivos do REPAssistive, facilitando o seu uso, principalmente quando educandos com NEE não alfabetizados realizam interações com o mesmo.

\subsection{Avaliação de Usabilidade}

A segunda parte da avaliação do REPAssistive foi executada tomando como base a aplicação de testes de usabilidade. Analisando os dados obtidos através dos surveys respondidos entre os educadores e os especialistas da área de interface gráfica, os resultados salientam que 7 entre as 10 heurísticas avaliadas, receberam $100 \%$ de aprovação. Assim, ao observar a Figura 5, é possível identificar a avaliação entre cada uma das heurísticas.

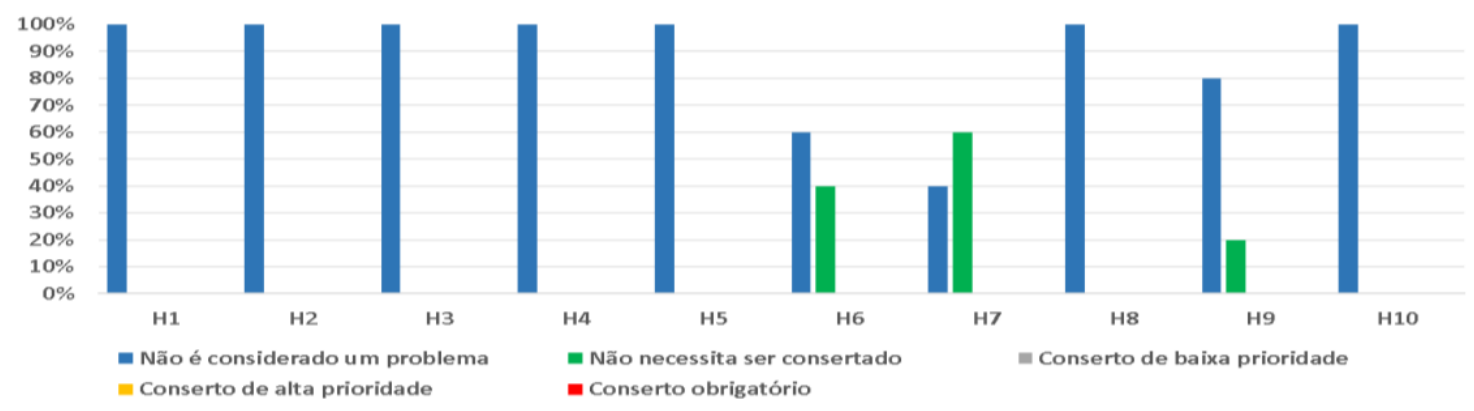

Figura 5. Resultados referentes a avaliação das heurísticas.

Diante dos dados apresentados, as heurísticas H1, H2, H3, H4, H5, H8 e H10 obtiveram como resposta o grau mínimo de acordo com os possíveis problemas detectados. Ou seja, foram classificadas com o grau de nível 1, caracterizando-se, na opinião dos avaliadores, como um grupo de heurísticas nas quais os seus conceitos estão bem aplicados e que não possuem dificuldades e contrariedades que possam acarretar em problemas durante a interação e o uso. Por outro lado, as heurísticas H6, H7 e H9 adquiriram satisfação de $60 \%, 40 \%$ e $80 \%$, respectivamente, associando-se a classificação de nível 2, na qual há um pequeno problema, mas que o mesmo "não necessita ser consertado". Acredita-se que esses resultados estejam relacionados a pequenas confusões onde os usuários ficaram com dúvidas sobre qual passo seguir após realizar uma ação no repositório, como também a restrição que determina o preenchimento de todas as informações de modo obrigatório no momento em que deseja-se realizar uma colaboração de um novo recurso.

\subsection{Limitações e Ameaças}

A pesquisa apresenta algumas ameaças à validade: $(i)$ as avaliações realizadas com um número pequeno de avaliadores; (ii) as avaliações efetuadas com apenas um método proposto; (iii) o perfil do grupo de educandos em simpatizar com jogos; (iv) a avaliação com os educandos foi realizada utilizando apenas o tipo de Deficiência Intelectual. 


\section{Considerações Finais}

Neste artigo é apresentado um repositório virtual, inicialmente, contendo recursos do tipo jogos educacionais digitais com conteúdos relacionados à linguagem e raciocínio lógico para o uso de educandos com NEE. Esse tipo de repositório ainda não existia com o foco, especificamente, em RED atribuídos a metodologia de ensino e aprendizagem dentro da educação especial. Agora, com o repositório desenvolvido, pode-se compartilhar as informações de diversos jogos de uma forma organizada e com uma busca estruturada por específicos atributos. Além do uso do manual do professor, que auxilia o educador na preparação da aula e contribui com um conjunto de informações pedagógicas.

Desta forma, o repositório fortalece o conhecimento de modo intuitivo, provocando maior desenvolvimento intelectual, educacional e profissional. Do mesmo modo que os jogos educacionais contidos no mesmo possibilitam a inclusão digital junto com a potencialização das habilidades cognitivas, despertando o interesse pela aquisição do saber entre os educandos com deficiência.

Para trabalhos futuros, pretende-se evoluir o repositório com base nas sugestões de melhorias apontadas pelos participantes nos processos de avaliações. Além de inserir diferentes tipos de RED, juntamente com o aumento no número de jogos educacionais de acordo com os variados tipos de necessidades educacionais específicas.

\section{Referências}

Carvalho, R. E. (2010). Removendo barreiras para a aprendizagem, v. 21. Rio de Janeiro.

Júnior, J. B. B. (2013). Portais educacionais e suas características: contribuições para o estado da arte. Holos, 3:111-129.

Lima, C. A., Hitzschky, R., de Brito, M. A., Oliveira, A. G., de Castro Filho, J. A., and Medeiros, M. D. (2016). Uma proposta de catalogação de recursos educacionais digitais utilizando tablets para o ensino de crianças com deficiência intelectual. Anais do Congresso Regional sobre Tecnologias na Educação, pages 510-517.

Nielsen, J. and Mack, R. L. (1994). Inspection Methods. New York.

Silva, C., Osmundo, M. L. F., Hitzschky, R. A., De Brito, M. A. F., De Castro Filho, J. A., and Medeiros, M. D. (2016). Processo de criação de um repositório educacional digital: Procedimentos de busca, seleção e categorização de recursos educacionais digitais (RED). Anais do Congresso Regional sobre Tecnologias na Educação, pages 427-437.

Silva, R. A., Paula, M. M. V., and Sarlas, L. M. V. (2011). Utilização de jogos para pessoas com necessidades educativas especiais: uma análise experimental. Simpósio Brasileiro de Informática na Educação (XXII), pages 456-464.

Valente, J. A., Freire, F. M. P., and Arantes, F. L. (2018). Tecnologia e Educação (passado, presente e o que está por vir). NIED/UNICAMP, Campinas-SP. 